\title{
ENTERAL AND PARENTERAL NUTRITION
}

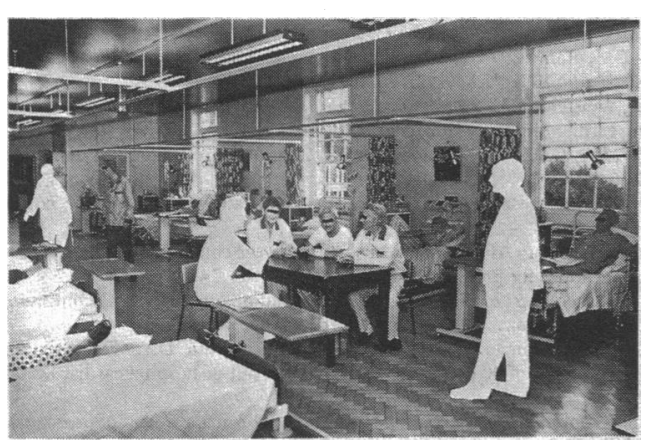

Patients in hospital have a high risk of nutritional disorders, a risk that rises with increasing length of stay. Some patients are admitted with an illness that has caused the problem. Others develop nutritional complications whilst undergoing treatment. Bistrian and Blackburn considered that $44 \%$ of general medical ${ }^{1}$ and $50 \%$ of general surgical patients ${ }^{2}$ in the wards of an American municipal hospital had some features of protein calorie malnutrition. Hill found that $26 \%$ of patients in the surgical wards of the Leeds General Infirmary were hypoalbuminaemic. ${ }^{3}$ Although minor degrees of protein calorie malnutrition do not appear to affect the outcome of surgical operation major nutritional disorders undoubtedly jeopardise recovery.

As many as $50 \%$ of patients on a general surgical ward may show some manifestation of protein calorie malnutrition. The incidence of protein calorie malnutrition rises in patients who stay in hospital for over two weeks. ${ }^{4}$

\section{Types of malnutrition in hospital patients}

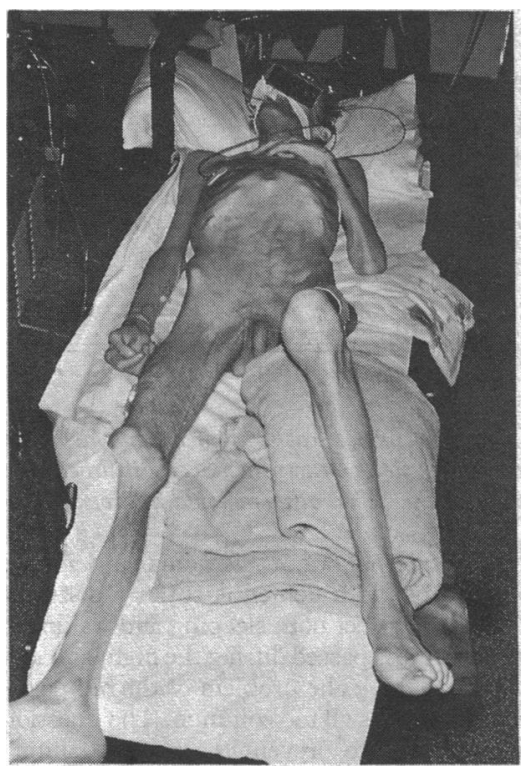

Surgical patients with septic complications tend to have a kwashiorkorlike malnutrition characterised by a low serum albumin concentration, muscle wasting, and water retention. On the other hand, medical patients tend towards marasmus. Most patients who develop a nutritional problem after operation have a mixed picture resulting from starvation, increased catabolism, and reduced anabolism. Malnutrition in surgical patients is accompanied by an increased risk of postoperative complications.

\section{Detecting malnutrition}

Some criteria of malnutrition in inpatients $10 \%$ Recent unintentional weight loss Body weight $<80 \%$ of ideal for height Serum albumin less than $30 \mathrm{~g} / \mathrm{l}$

Total lymphocyte count of less than $1 \cdot 2 \times$ $10^{6} / 1$
Protein calorie malnutrition resulting from a combination of sepsis and starvation induced by an intestinal fistula.

Many methods of detecting protein-calorie malnutrition have been advanced, varying from the sophisticated to the simple. Those requiring complex equipment, such as neutron activation analysis for measuring total body nitrogen, are research tools. Valuable information can be obtained from simpler measurements such as change in body weight, arm muscle circumference, and serum albumin concentration. These can, however, be difficult to interpret in the short term because of complicating factors such as water retention. Once malnutrition is detected treatment should be started to reverse it. Nutritional treatment will not be effective in the presence of active sepsis. The priority in such cases is to eliminate the septic focus. 


\section{Treating malnutrition}

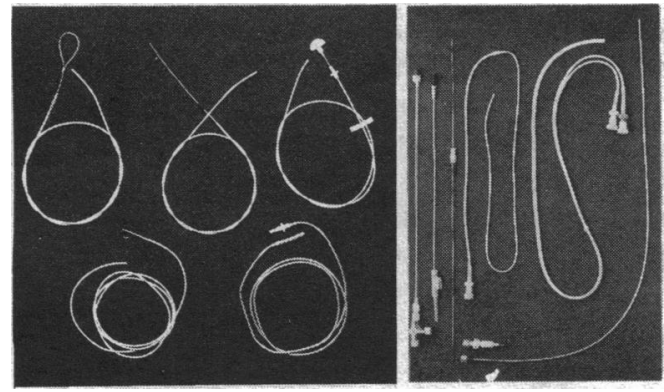

\section{Enteral nutrition}

\begin{tabular}{|l|}
\hline Indications for enteral nutrition \\
Unconsciousness \\
Neurological dysphagia \\
Oesophageal obstruction \\
Inflammatory bowel disease \\
Short bowel syndrome \\
Post-traumatic weakness \\
Postoperative weakness \\
Post irradiation weakness \\
Head and neck surgery \\
Chemotherapy \\
Burns \\
Old age \\
\hline
\end{tabular}
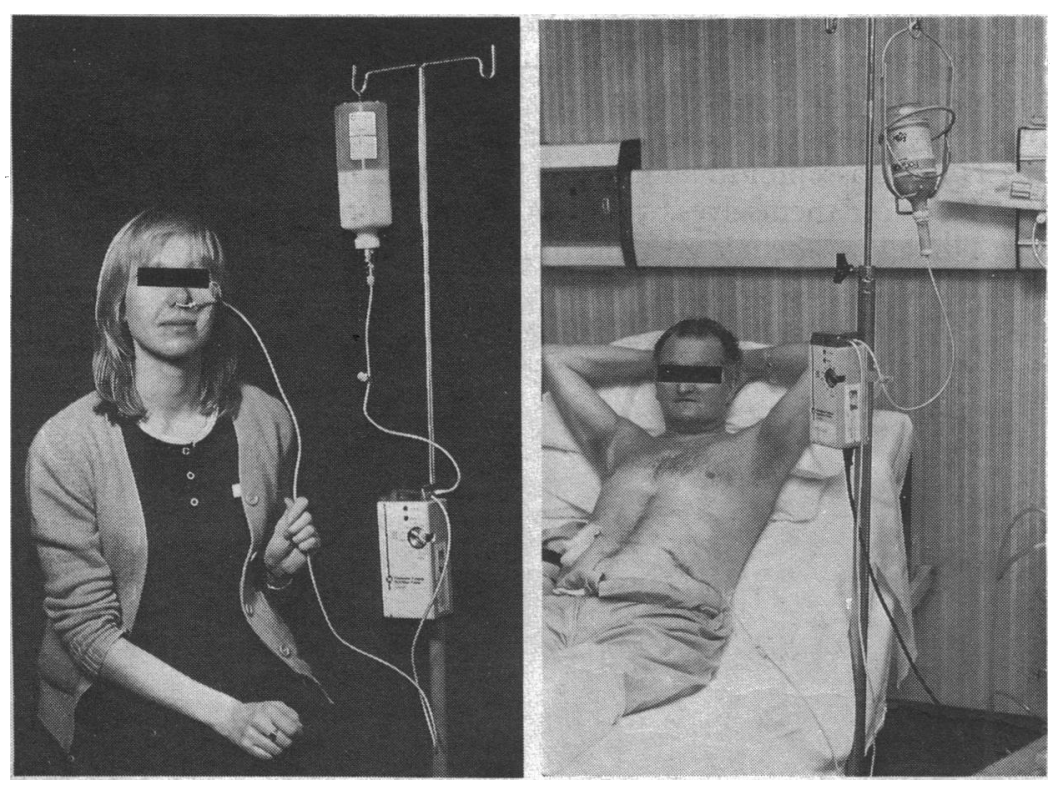

Enteral nutrition using chemically defined liquid regimens should be prescribed only when patients cannot eat normal food. Enteral nutrition can be total or supplemental. Supplemental feeding is used when a patient needs an easily taken nutrient preparation to supplement an inadequate intake of normal food. Total enteral nutrition is primarily indicated in patients who cannot eat or drink because of unconsciousness, partial obstruction or disease of the intestinal tract or inability to swallow because of neurological disorders. Some patients need enteral nutrition with a liquid diet because they cannot swallow solids or because of high losses from fistulas or stomas.

\section{Administration of total enteral nutrition}

In some patients who cannot eat because of dysphagia the normal diet may be liquidised and swallowed in the usual way. Alternatively a commercially prepared liquid food may be used. Patients who cannot swallow or those with continually high losses from stomas or fistulas will need tube feeding.

Modern feeding tubes are of fine bore and made of polyurethane or silastic. They are easily tolerated by the patient and can remain in position for long periods without damaging the oesophagus. Their fine bore precludes their use for the administration of liquidised food; only the commercially available chemically defined preparations can be easily administered through them. In patients with total oesophageal obstruction or upper intestinal fistulas the diet can be infused directly into the jejunum through a fine tube jejunostomy.

Left: fine bore nasogastric feeding tube in position with direct administration from container. Right: patient with gastric outlet obstruction and duodenal fistula being fed through a fine bore jejunostomy tube.

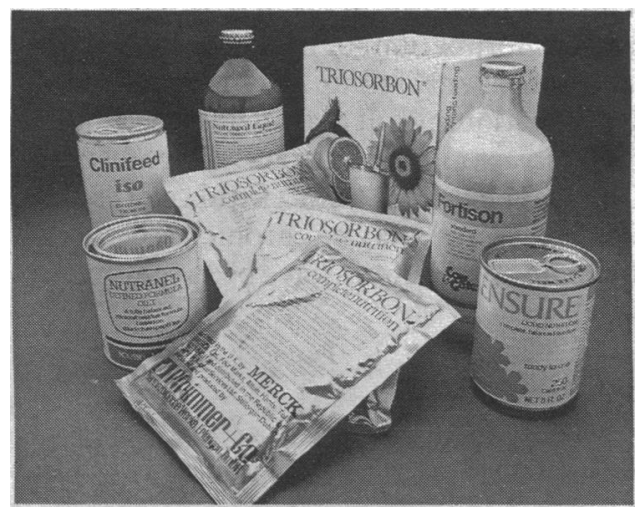

\section{Choice of enteral nutrient}

There is a wide range of enteral preparations, the principal differences between them being in the way the protein and energy are presented. Liquid whole protein regimens are cheaper and more palatable than those based on oligopeptides and amino acids.

The oligopeptide and amino acid preparations are alleged to be better absorbed, especially in patients with shortened or diseased bowel. But there is no good evidence that this is so, and the preparation of choice for routine use is a whole protein regimen. The energy content of the diet is offered as glucose, oligosaccharides, maltodextrin, corn syrup, medium chain triglycerides, sunflower oil, etc. Other essential nutrients, such as electrolytes, minerals, trace elements, and vitamins, are added in varying quantities depending on the preparation. Several diets do not contain 
Components of typical whole protein polymeric liquid enteral diet Water

Sodium caseinate Coconut oil

Calcium caseinate

Lecithin

Maltodextrin

Minerals

Trace elements

Corn oil

Vitamins

Palm oil

$500 \mathrm{ml}$ of this diet provides $20 \mathrm{~g}$ of protein $(3 \cdot 15 \mathrm{~g}$ of nitrogen), $500 \mathrm{kcal}$ $(2 \cdot 1 \mathrm{MJ})$

lactose and can be used in patients with lactose intolerance. Variations of the basic formula allow for increased energy and nitrogen provision or reduced sodium content.

Composition of an ideal enteral diet - An average patient will need $2000-3000 \mathrm{kcal}(8 \cdot 4-12 \cdot 6 \mathrm{MJ})$ and $10-15 \mathrm{~g}$ of nitrogen, corresponding to $60-90 \mathrm{~g}$ protein in 2-3 litres of fluid. The proportion of energy provided by fat should be about $30-40 \%$. The mixture should contain minerals, trace elements, and vitamins.

\section{Complications of enteral feeding}

Simply because an enteral regimen is being

\section{Complications}

Gastric retention

Aspiration

Nausea and vomiting

Diarrhoea

Dehydration
Hyperosmolar coma

Hyperglycaemia

Tube misplacement

Oesophageal erosions

Infection administered into the gastrointestinal tract it cannot be assumed that the treatment is relatively free of complications. Patients receiving this treatment are at risk from aspiration, vomiting, diarrhoea, and disturbances of metabolism and water balance. Additionally, careless handling of the regimen can result in it becoming infected.

\section{Ambulatory home enteral nutrition}

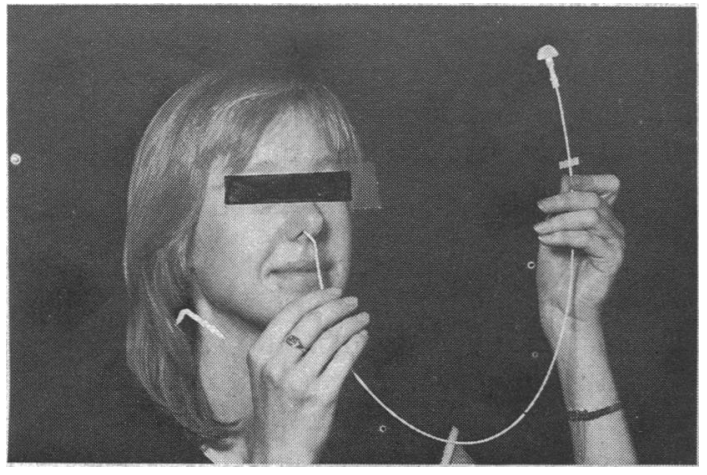

In some patients the need for nutrients is so high that continuous administration is necessary. Others can only cope with their requirements by prolonged nutrition. Such patients can be taught to pass feeding tubes themselves and assemble their infusion for overnight administration or alternatively feed themselves by continuous infusion using a portable pump. This allows them to go home from hospital and resume a more normal life, including going to work.

Patient inserting fine bore feeding tube into stomach for continuous overnight intragastric infusion.

\section{Parenteral nutrition}

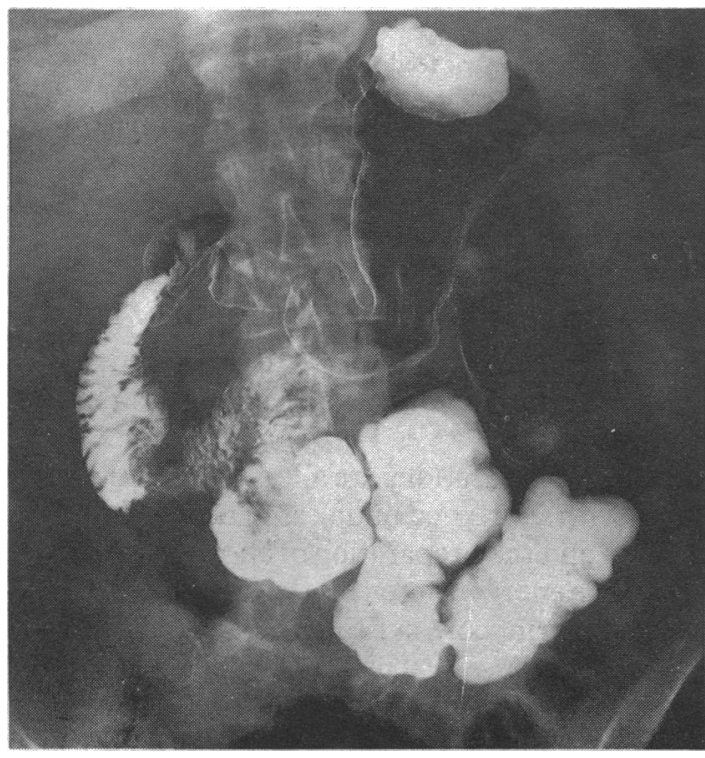

Intravenous administration of nutrients is indicated when patients cannot be fed by mouth, by nasogastric intubation, or by jejunostomy. Such patients can be said to be in a state of "intestinal failure." This condition may be defined as "the reduction of functioning gut mass below the amount necessary for adequate digestion and absorption of nutrients." ${ }^{6}$ Intestinal failure may be acute and reversible, as for example until a fistula closes or a segment of short bowel adapts. Alternatively it may be chronic, as in cases of short bowel, where virtually all ileum and jejunum have been removed.

Barium meal in a case of short bowel syndrome. Duodenocolic anastomosis after total excision of small bowel. 
Principal causes of intestinal failure

Reduction in absorbtive surface-short bowel

- Premature loss of enteric content-intestinal fistula

- Disorder of peristalsis—chronic idiopathic intestinal pseudo-obstruction

- Parenchymal disease of small bowel-Crohn's, radiation enteritis

\section{Technique of parenteral nutrition}

Access to the circulation for intravenous feeding can be by a peripheral vein. But even when isotonic solutions are used, the veins tend to thrombose, making this method one for only short term use. For long term intravenous feeding the catheter is best introduced into a major vein such as the subclavian, the tip being advanced until it lies in the superior vena cava. The remainder of the catheter is tunnelled in the subcutaneous tissues to emerge on the anterior chest wall.
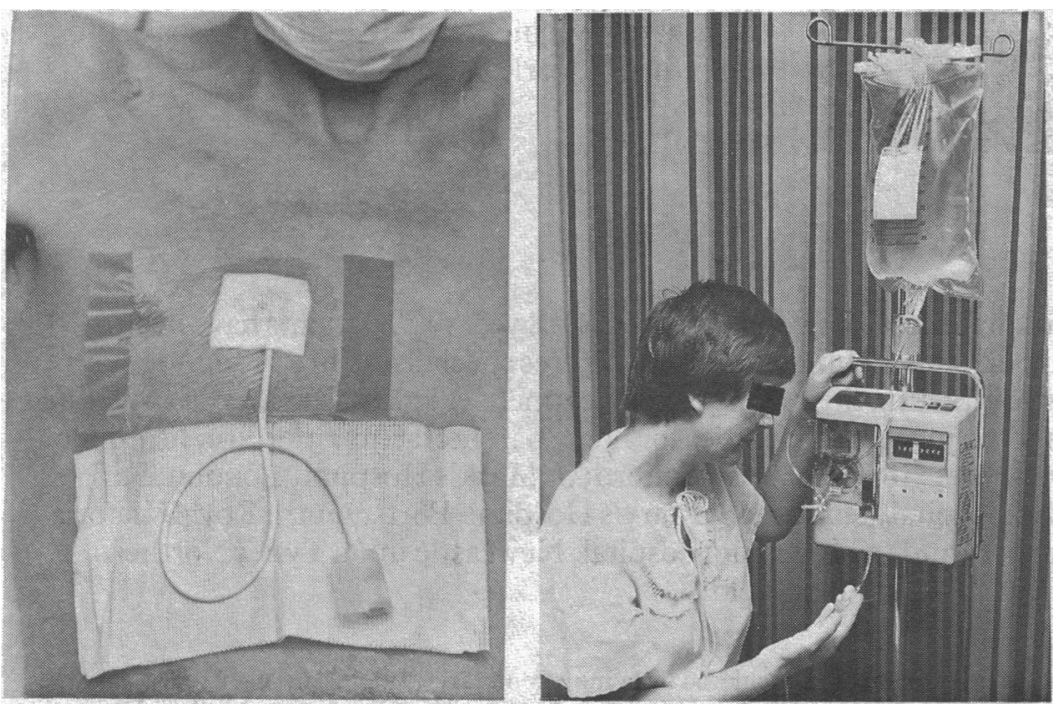

With careful attention to asepsis and the use of antiseptic dressings infection can be prevented and the catheters can remain in situ indefinitely. Although in the early stages parenteral nutrition is given throughout the day, once a patient stabilises the feed may be given just during the night. During the day the catheter can be filled with heparin, thereby allowing the patient to move around normally.

Nutrients are administered to the patient from a 3 litre bag which is filled in the pharmacy under sterile conditions. A regular rate of infusion is ensured by using a constant volume infusion pump which incorporates alarms to warn of air in the infusion system and changes in the flow rate.

Left: silastic catheter running in subcutaneous tunnel from subclavian vein to emerge on chest wall. Right: constant volume infusion pump for continuous administration of nutrient. Three litre bag contains $24 \mathrm{~h}$ requirements of water, electrolytes, amino acids, calories, trace elements, and vitamins.

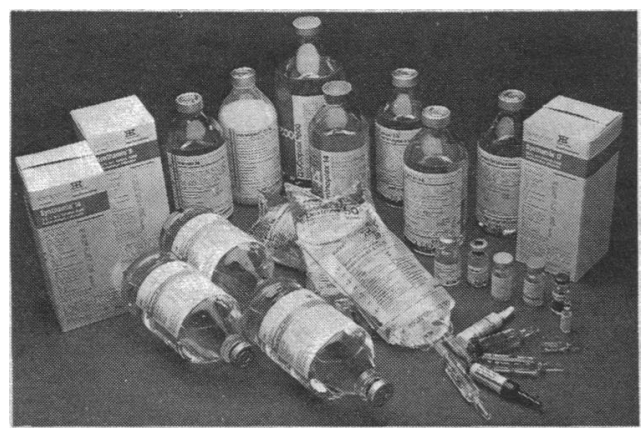

Intravenous amino acid solutions, glucose, fat, vitamin, and trace metal supplements used in total parenteral nutrition.

Some complications of total parenteral nutrition

Catheter infection

Catheter fracture

Catheter tip malposition in pleura or subclavian artery

Catheter induced venous thrombosis

Air embolism

Fluid overload

\section{Nutrients used in parenteral feeding}

The regimen used is broadly tailored to an individual's requirements. A stable patient with intestinal failure usually requires about $2500 \mathrm{kcal}$ ( 10.5 $\mathrm{MJ}$ ) of energy and $12 \mathrm{~g}$ of nitrogen as crystalline amino acids in $2500 \mathrm{ml}$ of fluid. Energy is provided using glucose and lipid emulsion. In the United Kingdom the latter is a soya bean oil emulsion which seems to have the same properties as chylomicrons. In patients in hospital lipid usually provides about $30 \%$ of the calories infused.

Amino acid provision includes all the essential amino acids, and a wide range of non-essential ones. The ratio of amino acids one to another-the aminogram-usually approximates that of a high quality protein such as egg albumin.

Mixed into the bag with the above are the normal daily requirement of electrolytes, trace elements, and vitamins. Treatment with parenteral nutrition continues until the underlying condition has resolved and enteral nutrition can be reintroduced.

\section{Complications of parenteral nutrition}

The principal complication of parenteral nutrition is infection of the intravenous feeding catheter which can produce septicaemia. If this happens the catheter must be removed.

Thrombosis of the vessel into which the infusion is being delivered can occur. Extravasation of the infused fluid due to misplacement of the catheter tip is preventable by screening at the time of placing the catheter.

Metabolic problems such as hyperglycaemia can arise from infusion of the glucose load, although this usually settles as the patient's body adapts to this form of treatment. In the long term trace element deficiencies can create problems but these are preventable by careful monitoring. 


\section{Ambulatory home parenteral nutrition}

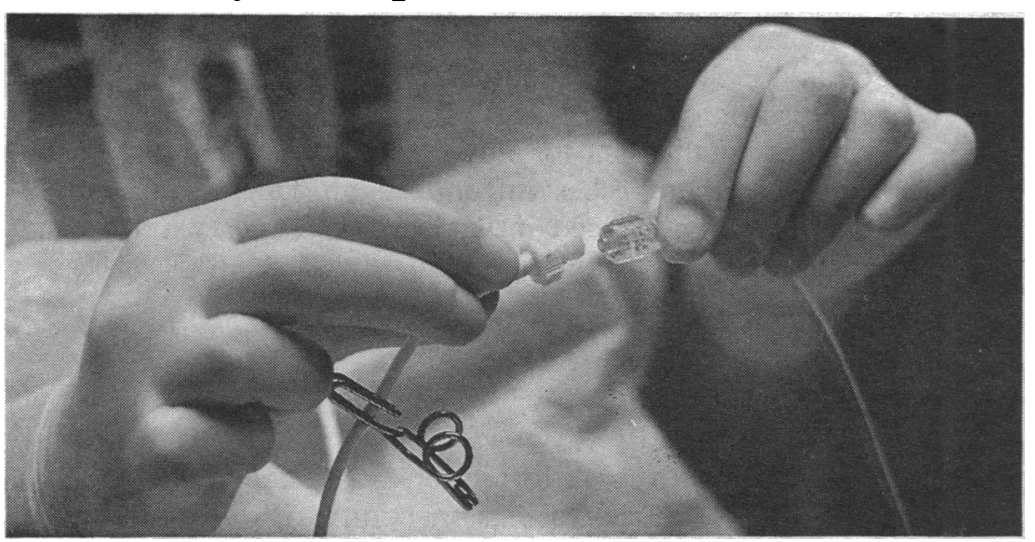

In patients in whom restoration of enteral nutrition is likely to be delayed or in those committed to lifelong support by parenteral feeding the advantages of home parenteral nutrition should be considered. Patients can be taught the techniques of catheter care and intravenous infusion. This enables them to leave hospital and return to the community. Most of these patients, by feeding themselves overnight, can live an active social life and return to work.

Home parenteral nutrition patient connecting herself to infusion.

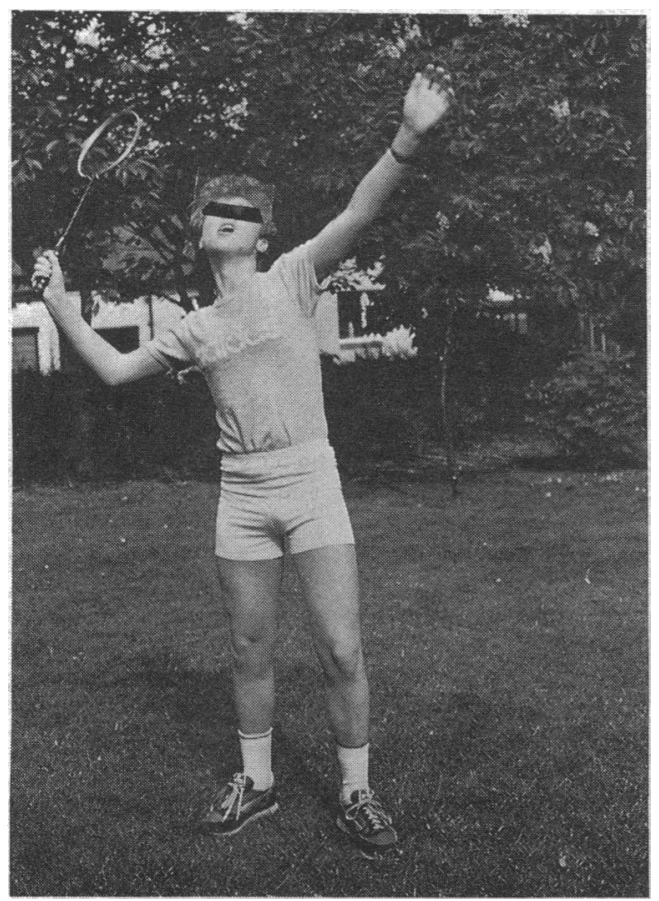

In the United Kingdom over the last 8 years 170 patients have been trained in this technique, most in centres specialising in its use. A few are now completing their fifth year of treatment. Principal centres in the United Kingdom providing facilities for home parenteral nutrition are, in order of experience, Hope Hospital, Salford; St Mark's Hospital, London; King's Cross Hospital, Dundee; St Mary's Hospital, Portsmouth; Royal Victoria Infirmary and the Freeman Hospital, Newcastle upon Tyne; Northern General Hospital, Sheffield.

A good quality of life can be achieved by home parenteral nutrition. This fit 16 year old is in his second year on home parenteral nutrition after duodenocolic anastomosis occasioned by total loss of the small bowel resulting from volvulus.

1 Bistrian BR, Blackburn GL, Vitale J, Cochran D, Nayla J. Prevalence of malnutrition in general medical patients. FAMA 1976;235:1567-70.

2 Bistrian BR, Blackburn GL, Hallowell E, Heddle R. Protein status of general surgery patients. FAMA 1974;230:858-60.

3 Hill GL, Pickford I, Young GA, et al. Malnutrition in surgical patients: an unrecognised problem. Lancet 1981;i:689-92.

4 Weinsier RL, Butterworth CE. Handbook of clinical nutrition ch 1. St Louis: CV Mosby, 1981:6.

5 Fleming CR, Remington M. Nutrition and the Surgical Patient. In: Hill GL, ed. Clinical surgery international. London: Churchill Livingstone, 1981:219-35.

6 Silk DBA. Nutritional support in hospital practice. Oxford: Blackwell, 1983.

Professor Miles Irving, MD, FRCS, is professor of surgery, Hope Hospital, University of Manchester.

How common is magnesium deficiency and what treatment is advised if a patient is found to be deficient?

Deficiency of this important intracellular and skeletal component is reflected in the serum concentration and is usually associated with obvious disease or particular drug treatment. The usual clinical features of hypomagnesaemia are paraesthesiae, cramp, and tetany, symptoms that may be due more to the invariably associated hypocalcaemia. Symptomatic disease is probably rare in general practice but the prevalence is not known with certainty as measurements of serum magnesium concentrations are infrequently requested. Some indication of its prevalence in the population seeking medical advice is given by the private general diagnostic clinic survey of Jackson and Meier, ${ }^{1}$ whose screening of 5100 subjects showed 18 patients with hypomagnesaemia ( $>3$ SD below mean), six of whom were diabetic, seven taking diuretics, and five had no explained cause.

Gastrointestinal and renal causes account for almost all magnesium deficiency ${ }^{2}$ but inadequate dietary intake alone is a relatively unimportant cause. In malabsorptive states hypomagnesaemia occurs in $\mathbf{4 0} \%$ of cases. Loss of intestinal secretions is an important cause: severe diarrhoea, purgative abuse, gastrointestinal fistulae, intestinal resection, or bypass surgery and conditions such as Crohn's disease and ulcerative cholitis are all associated with hypomagnesaemia. Urinary loss of magnesium is a feature of some renal tubular disorders but rarely of glomerular disease. Drug induced renal losses are seen in some patients taking thiazide and loop diuretics and also with aminoglycoside antibiotics. Acute urinary loss occurs in normal subjects and in alcoholic subjects after administration of ethanol. Hypomagnesaemia is common in alcoholics, occurring in $30 \%$ of admitted alcoholic patients and in $86 \%$ of those with delirium tremens. Mild hypomagnesaemia is best treated with oral magnesium glycerophosphate, which is reasonably well tolerated. Other magnesium salts and magnesium hydroxide may also be used but are poorly absorbed and cause diarrhoea. Hypomagnesaemia with severe symptoms should be treated parenterally with magnesium sulphate, care being taken to administer it separately from any calcium supplements with which it would cause precipitation of calcium sulphate.-K O LEWIS, principal biochemist, Birmingham.

1 Jackson CE, Meier DW. Routine serum magnesium analysis. Ann Intern Med 1968;69:743-8. 2 Brenton DP, Gordon TE. Fluid and electrolyte disorders: magnesium. Br f Hosp Med 1984;32: 68-9.

3 Levine BS, Coburn JW. Magnesium, the mimic/antagonist of calcium. N Engl f Med 1984;310: 1253-4. 